\title{
Recurrent Auricular Pseudocyst: Successful Treatment Using a Dental Silicon Cast
}

\author{
Yusuke Yoshioka ${ }^{a}$ Takeshi Namiki ${ }^{a}$ Tsukasa Ugajin ${ }^{a}$ Hiroo Yokozeki ${ }^{a}$ \\ Atsushi Tanaka ${ }^{b}$ \\ aDepartment of Dermatology, Graduate School of Medical and Dental Sciences, Tokyo \\ Medical and Dental University, Tokyo, Japan; ${ }^{b}$ Department of Dermatology, Kameda \\ Medical Center, Chiba, Japan
}

Keywords

Recurrent $\cdot$ Auricular $\cdot$ Pseudocyst $\cdot$ Dental silicon cast $\cdot$ Treatment

\begin{abstract}
Auricular pseudocysts (APs) are benign intercartilaginous cysts at the auricle. Various therapeutic options have been used to treat APs, but they frequently recur in usual clinical practice. Here, we report a case of AP successfully treated using a silicon cast. A 65-year-old male presented with a cyst on his right auricle. Although a local injection of steroid was administered, the cyst had recurred. A dental silicon cast was applied after removal of the contents of the cyst by suction. There was no recurrence of the cyst 6 years after that treatment. Dental silicon casts are a novel technique for an appropriate fixation over auricles in the treatment of APs and had an effective therapeutic result in our case.
\end{abstract}

\section{Introduction}

Auricular pseudocysts (APs) are benign intercartilaginous cysts at the ear. APs usually affect the upper half of the auricle and contain viscous fluid in an intercartilaginous cavity [1-3]. Various therapeutic options including surgical treatments and steroid injections have been used to treat APs $[3,4]$, but they frequently recur in usual clinical practice. Dental silicon casts have been used for the treatment of various facial deformities such as rhinoplasty due to their ability to fit various curved body surfaces. Here, we report a case of an AP successfully treated using a silicon cast. 
Fig. 1. A $20 \times 20 \mathrm{~mm}$ cyst in the patient's right auricle had a relatively clear margin, no redness, and no swelling.
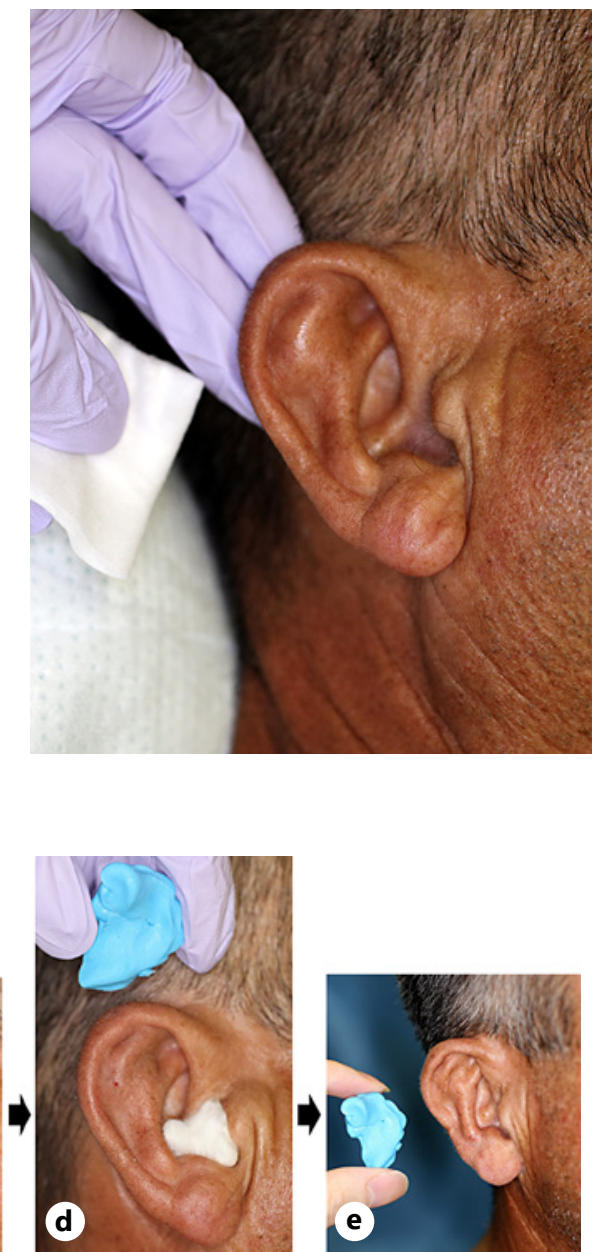

d)
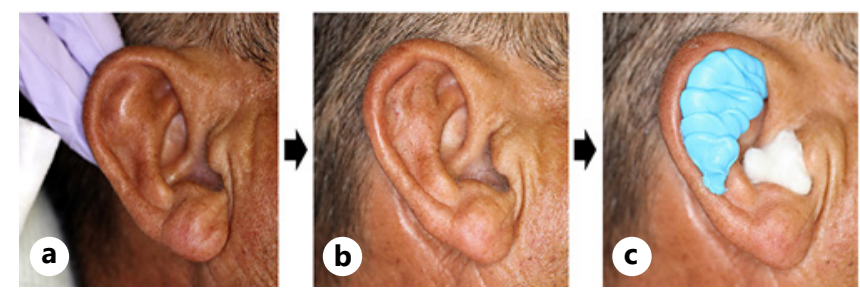

Fig. 2. Application of a silicon cast on the patient's auricle. a Before suction of the contents in the cyst. $\mathbf{b}$ After suction of the contents in the cyst. c Mold making of the silicon cast at the auricle. $\mathbf{d}$ Completed silicon cast after mold making. Temporarily, the silicon cast was removed from the auricle for comparison between the silicon cast and the shape of the patient's auricle. e One month after the compression with a silicon cast. There was no recurrence of the AP. AP, auricular pseudocyst.

\section{Case Report}

A 65-year-old male presented with a subcutaneous cyst on his right ear. The patient had recognized the swelling on his right ear 2 weeks before. Although a local injection of steroid was administered after removing the contents in the cyst by suction at his first visit, the cyst had recurred. The patient visited the outpatient clinic again. Physical examination revealed a $20 \times 20 \mathrm{~mm}$ cyst at his right auricle. The margin of the cyst was relatively clear without redness and swelling around the cyst (Fig. 1). Laboratory investigations revealed no abnormal values regarding the cyst. From both the clinical presentation and the clinical course, the diagnosis of a recurrent AP was made. Initially, we planned to use steroid injections and/or surgical removal of the cyst. However, we excluded a second local injection of steroid as the next therapy due to the failure of the injection at his initial visit and also excluded surgical removal due to no indication for surgical removal because the contents of the cyst were mostly accumulated liquid. Next, we considered using a surgical bolster and/or clip compression dressing but the uneven pressure exerted by those methods would not be preferable. On the

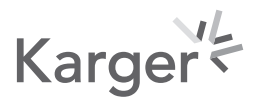



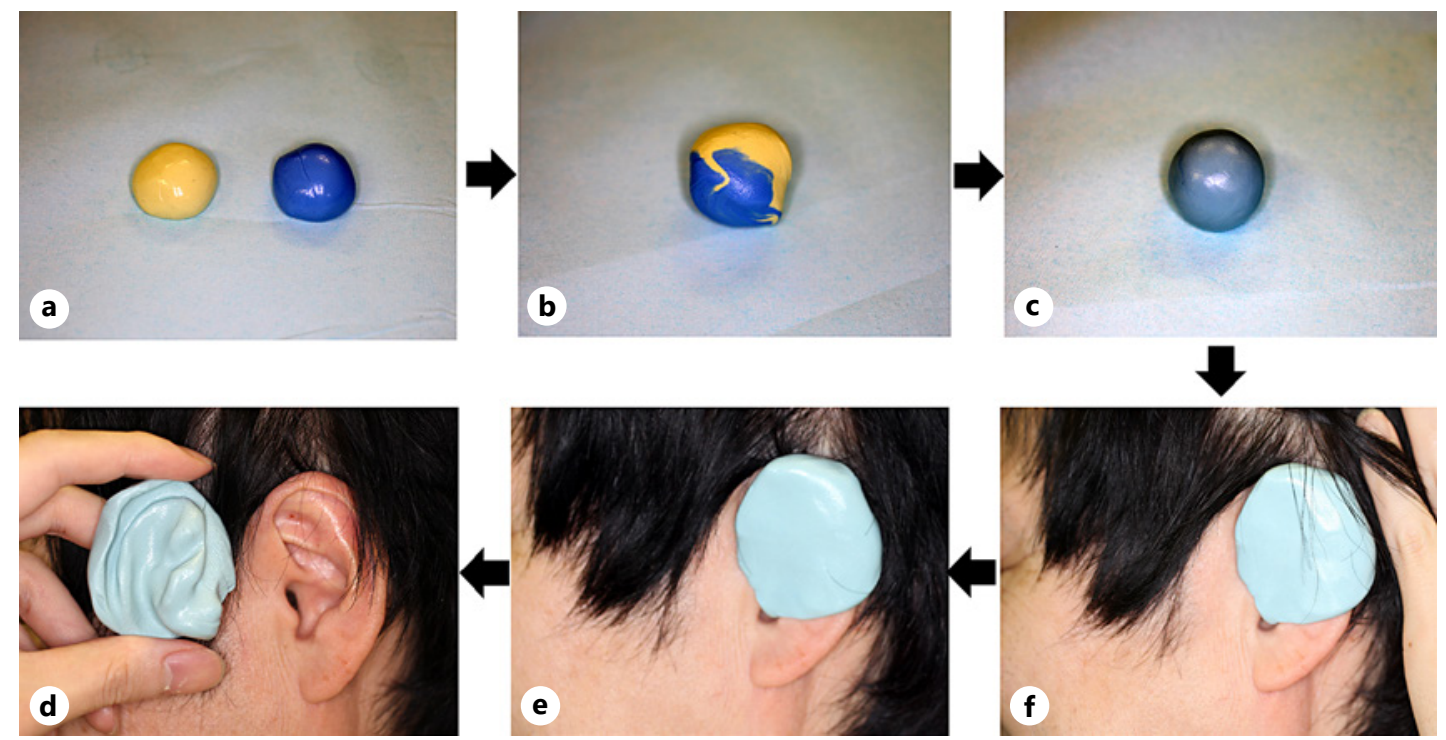

Fig. 3. A brief procedure of the application of a silicon cast for the treatment of an AP: a prepare similar amounts of the 2 different pastes. b Combine those 2 pastes. c mix well until the color of the mixed paste changes to light blue. $\mathbf{d}$ adjust the shape of the mixed paste to the patient's auricle. $\mathbf{e}$ harden the mixed paste for 1 min $\mathbf{f}$ the completed mixed paste with the shape of the auricle. AP, auricular pseudocyst.

other hand, compression by silicon has been widely used in several plastic surgical procedures such as rhinoplasty. Finally, we came up with the idea to use dental silicon to generate even pressure after removing the contents of the AP by suction. In this patient, the contents in the cyst were removed and a dental silicon cast was then shaped to fit his auricle (Fig. 2a-d). After 1 month of compression, the AP was cured without recurrence (Fig. 2e), and 6 years after that treatment, there has been no recurrence.

\section{Discussion/Conclusion}

APs are generally believed to occur in intercartilaginous cavities and retain fluids from the surrounding tissues. APs are devoid of an epithelial lining at the inner surface of the cyst and are called "pseudocysts" [1]. Various therapies have been attempted to reduce the recurrence of APs. The most common treatments aim to remove APs or reduce their recurrence with minimal damage to the surrounding skin and cartilaginous tissues. Although simple aspiration, the injection of steroid, and aspiration with bolstered pressure sutures are effective in some cases, an appropriate pressure will dramatically enhance the success of those treatments. Pressures after those treatments may minimize the chance of accumulating fluid and might result in an improved therapeutic outcome. Several techniques to exert pressure on ears have been developed for use after those treatments. Some techniques, such as surgical bolstered sutures, are invasive, but other techniques are noninvasive $[1,5]$. Critical points for the successful application of pressure are even and appropriate forces on the uneven surface of the auricle. In order to fulfill this requirement, we decided to use a dental silicon cast from the better performance of silicon casts used in the field of plastic surgery such as rhinoplasty. We took advantage of the "Imprint Bite (3M, St. Paul, MN USA)" for this purpose due to the short time required for the hardening of silicon. Each step of the procedure for the use of the "Imprint Bite" is shown briefly in Figure 3. Compression by a dental silicon 
cast has several advantages over previous methods: firm fixation over auricles is possible using a dental silicon cast and an adhesive tape, they are noninvasive and it takes a brief time to accomplish the entire procedure. Dental silicon casts are a novel technique for an appropriate fixation over auricles to treat APs and had a better therapeutic result in our case.

\section{Statement of Ethics}

The authors have obtained written informed consent from the patient for the publication of this case report. This work was conducted in accordance with the Declaration of Helsinki.

\section{Conflict of Interest Statement}

The authors have no conflicts of interest to declare.

\section{Funding Sources}

The authors have no funding source to declare.

\section{Author Contributions}

Y.Y. and T.N. drafted the manuscript. T.U., H.Y., and A.T. revised the manuscript critically. Y.Y., T.N., T.U., and A.T. were involved in the conception of this manuscript. All the authors gave final approval of the latest version of this manuscript.

\section{References}

1 Schulte KW, Neumann NJ, Ruzicka T. Surgical pearl: the close-fitting ear cover cast -a noninvasive treatment for pseudocyst of the ear. J Am Acad Dermatol. 2001 Feb;44(2):285-6.

2 Paul AY, Pak HS, Welch ML, Toner CB, Yeager J. Pseudocyst of the auricle: diagnosis and management with a punch biopsy. J Am Acad Dermatol. 2001 Dec;45(6 Suppl l):S230-2.

3 Miyamoto H, Oida M, Onuma S, Uchiyama M. Steroid injection therapy for pseudocyst of the auricle. Acta Derm Venereol. 1994 Mar;74(2):140-2.

4 Grigoryan KV, Ingraffea A. Surgical deroofing procedure for the treatment of an auricular pseudocyst. Dermatol Surg. 2015 Oct; 41(10):1198-200.

5 Wu MY, Chen CB, Chen YH, Chang C, Chan KC. Sandwich compression with rubbery tourniquet sheets and cotton balls for auricular pseudocyst. Laryngoscope. 2018 Jul;128(7):1653-7.

\section{Karger' ${ }^{\prime \prime}$}

